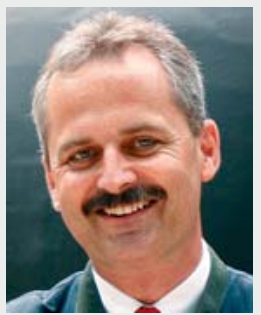

Florian Gebhard

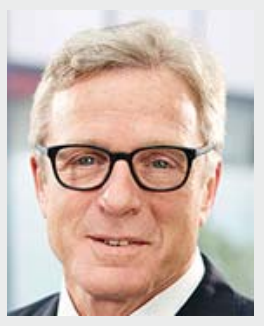

Michael J. Raschke

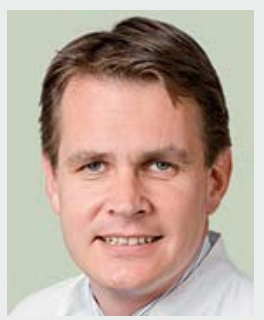

Ulrich Stöckle

\title{
Sehr geehrte Leserin, sehr geehrter Leser,
}

das aktuelle OP-Journal widmet sich der perioperativen Infektion, einem gefürchteten, aber leider gar nicht so seltenen Begleiter der operativen Frakturversorgung und Endoprothetik.

Auch wenn hohe Hygienestandards in operativen Abteilungen und auf chirurgischen Stationen etabliert sind, lassen sich perioperative Infektionen leider nie komplett vermeiden. Zumindest teilweise sind sie auch bereits durch das Unfallereignis im Sinne von offenen Frakturen und/oder erheblichem Weichteilschaden bedingt. In jedem Fall bedarf die perioperative Infektion aber einer konsequenten, adäquaten Therapie.

Aktuell werden wir auch vor neue Herausforderungen gestellt: Die Resistenzentwicklung der Bakterien schreitet schneller voran als die pharmazeutische Entwicklung neuer wirksamer Antibiotika und die Globalisierung und Reisefreudigkeit unserer Patienten erfordert die Isolierung von Rückkehrern und die Behandlung von bisher in unseren Breiten nicht heimischen Keimen.

Gerade unter diesen Umständen gewinnen die adäquate Prophylaxe, perioperative Hygiene und chirurgische Therapie einen neuen, oder besser, ihren alten, Stellenwert zurück.

Namhafte Autoren, jeweils Spezialisten auf ihren Fachgebieten, haben den aktuellen "State of the Art“ in ihrem Bereich zusammengestellt und mit eindrücklichen Bildern aus Klinik, OP und Labor illustriert.

Der Stellenwert der plastischen Chirurgie wird dabei ebenso beleuchtet wie die Infektion im Fachgebiet der Mund-Kiefer-Gesichtschirurgie. Der aktuelle Stand der Technik und Forschung hinsichtlich antibiotikabeschichteter Implantate als Infektprophylaxe ist in einem eigenen Artikel hervorgehoben.

Neben den zahlreichen Artikeln aus der klinischen Praxis möchten wir in diesem Heft auch die Sonikation als neues Diagnostikum vorstellen und deren Einsatz und Nutzen erläutern. Der hochaktuelle Artikel zur „Antibiotic Stewardship“ lehrt uns den sinnvollen Umgang mit Antibiotika, wie er zunehmend auch gefordert wird.

Herrn PD Dr. Tobias Kraus danken wir für die Koordination dieser Ausgabe.

Verbunden mit dem Dank an alle Autoren wünschen wir Ihnen beim Lesen dieses Heftes viel Vergnügen und insbesondere auch Informationsgewinn. Wir sind uns sicher, dass Sie das eine oder andere Neue entdecken werden.

Mit freundlichen Grüßen

Ulrich Stöckle

Florian Gebhard

Michael J. Raschke

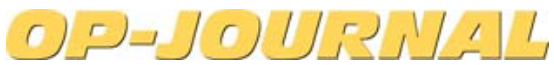

mitbegründet von

Prof. Dr. med. Günther Hierholzer

Prof. Dr. med. h. c. mult. Siegfried Weller

Herausgeber

AOTrauma Deutschland e. V.

Präsident: Prof. Dr. med. Michael J. Raschke, Münster
Schriftleiter

Prof. Dr. med. Florian Gebhard, Ulm

Prof. Dr. med. Michael J. Raschke, Münster

Prof. Dr. med. Ulrich Stöckle, Tübingen

Beirat

Priv.-Doz. Dr. med. Hermann Josef Bail, Nürnberg

Prof. Dr. med. Karl-Heinz Frosch, Hamburg

Priv.-Doz. Dr. med. dent. Alexander Gröbe,

Hannover
Prof. Dr. med. Marius Johann Baptist Keel, FACS, Bern, Schweiz

Prof. Dr. med. Dr. h. c. Edgar Mayr, Augsburg Prof. Dr. med. Michael Nerlich, Regensburg Prof. Dr. med. Hans-Christoph Pape, Zürich Dr. med. Michael Plecko, Graz, Österreich Prof. Dr. med. Klaus Schaser, Dresden Alexander Motzny, Tübingen 